\title{
Spectrum sensing and resource allocation for multicarrier cognitive radio systems under interference and power constraints
}

\author{
Sener Dikmese ${ }^{1^{*}}$, Sudharsan Srinivasan ${ }^{1}$, Musbah Shaat ${ }^{2}$, Faouzi Bader ${ }^{3}$ and Markku Renfors ${ }^{1}$
}

\begin{abstract}
Multicarrier waveforms have been commonly recognized as strong candidates for cognitive radio. In this paper, we study the dynamics of spectrum sensing and spectrum allocation functions in cognitive radio context using very practical signal models for the primary users (PUs), including the effects of power amplifier nonlinearities. We start by sensing the spectrum with energy detection-based wideband multichannel spectrum sensing algorithm and continue by investigating optimal resource allocation methods. Along the way, we examine the effects of spectral regrowth due to the inevitable power amplifier nonlinearities of the PU transmitters. The signal model includes frequency selective block-fading channel models for both secondary and primary transmissions. Filter bank-based wideband spectrum sensing techniques are applied for detecting spectral holes and filter bank-based multicarrier (FBMC) modulation is selected for transmission as an alternative multicarrier waveform to avoid the disadvantage of limited spectral containment of orthogonal frequency-division multiplexing (OFDM)-based multicarrier systems. The optimization technique used for the resource allocation approach considered in this study utilizes the information obtained through spectrum sensing and knowledge of spectrum leakage effects of the underlying waveforms, including a practical power amplifier model for the PU transmitter. This study utilizes a computationally efficient algorithm to maximize the SU link capacity with power and interference constraints. It is seen that the SU transmission capacity depends critically on the spectral containment of the PU waveform, and these effects are quantified in a case study using an 802.11-g WLAN scenario.
\end{abstract}

Keywords: CR; OFDM; FBMC; Filter bank; Spectrum sensing; Energy detector; Spectrum utilization; Loading algorithms; Multicarrier

\section{Introduction}

One of the major challenges in cognitive radio (CR) operation is to utilize the available whitespace with minimal interference to the primary or prioritized secondary transmission systems [1]. Several spectrum sensing techniques have been proposed, e.g., in [2-5] to facilitate CR operation. Especially, energy detector-based spectrum sensing algorithms have been widely considered due to low computational complexity. On the other hand, the fading channel capacity has already been studied from an information theoretic perspective, e.g., in [6,7] in terms of resource allocation. Recently, the secondary

\footnotetext{
* Correspondence: sener.dikmese@tut.fi

1 Department of Electronics and Communications Engineering, Tampere University of Technology, P.O. Box 692, Tampere 33101, Finland Full list of author information is available at the end of the article
}

user (SU) capacity has been widely studied. The SU channel capacity for additive white Gaussian noise (AWGN) channels under different power constraint is studied in [8]. The effect of various types of fading channels on the CR capacity has been studied in [9] under optimal power allocation strategy for the CR and subjected to an interference power constraint at the coexisting primary. Further, [10] discusses the effects of peak power and average interference power constraints on the outage capacity. In [11], the ergodic capacity, the delay-limited capacity, and the outage capacity of the CR in block-fading channels under spectrum sharing are discussed.

In this paper, we investigate two important features of the cognitive radio. We begin with the spectrum sensing function and later study the spectrum utilization 
function implementing optimized resource allocation under power and interference constraints. Instead of elaborate spectrum sensing techniques, such as cyclostationary and eigenvalue-based methods [2,3], energy detector-based spectrum sensing is utilized. This is motivated by subband-based energy detector's capability to implement the needed spectrum analysis functions for identifying the available spectral slots and for estimating the signal-to-interference-plus-noise (SINR) ratios at subcarrier level for resource allocation purposes.

For a CR system, multicarrier modulation techniques are generally better suited as they are spectrally more efficient than single carrier systems and have the flexibility to allocate resources to the available spectral gaps and among different users to maximize system throughput. There are various ways of improving the spectral containment of multicarrier waveforms, including methods to suppress the strong side lobes of the orthogonal frequency-division multiplexing (OFDM) spectrum [12-14]. Filter bank multicarrier (FBMC) is another multicarrier modulation scheme which has significantly reduced spectrum leakage compared to the cyclic prefix-based OFDM systems [15]. Also, the analysis filter bank (AFB) module of an FBMC receiver can be easily used for spectrum analysis purposes [15-22].

This paper includes a brief summary of our earlier studies concerning simple energy detection-based wideband multichannel spectrum sensing techniques for identifying the spectrum holes, considering the $2.4-\mathrm{GHz}$ ISM band as a case study. We apply an AFB-based energy detector, which averages the subband sample energies. By this way, multiple center frequencies, bandwidths, and multiple spectral gaps can be identified rapidly, efficiently, and flexibly for potential use by the CR. A similar fast Fourier transform (FFT)-based scheme is considered as a reference.

At the resource allocation stage, the transmit power of the subcarriers must be adjusted according to the channel state information (CSI) and the location of subcarriers with respect to the primary user's (PU) spectrum. In [23], an optimal and two sub-optimal power loading algorithms are developed. These algorithms use Lagrange formulation which maximizes the downlink capacity of the CR keeping the interference to the primary transmission below a threshold, without considering the total power constraint. In [23,24], the spectral hole and the signal-to-noise (SNR) are fixed to simplify the model. In [25], a low-complexity suboptimal algorithm is proposed. The algorithm gives maximum power to each subcarrier based on the results from conventional water filling and then modifies these values by applying power reduction algorithm in such a way that the interference constraint is satisfied. In $[23,25]$, the used signal models are closer to the ideal signal model, e.g., assuming fixed spectral hole bandwidth, instead of a realistic system model. In reality, the spectral hole bandwidth varies with the SNR. A proper system model should include also a practical power amplifier model. Our study focuses on the missing aspects of these studies.

The optimal solution which maximizes the CR link capacity under both transmission power and interference constraints requires high computational complexity, and it is unsuitable for the practical applications. Low complexity algorithms are proposed in $[25,26]$. However, in these methods, the interfered subcarriers are deactivated without considering optimized power and bit loading based on each subcarrier's SINR. Such optimization can be carried out using the power interference (PI) algorithm $[27,28]$. The resource allocation method utilizes the results of spectrum sensing in an efficient way, so there is interdependence between the spectrum sensing and spectrum allocation functions, which has not been addressed in earlier work. We study this interdependence, focusing on its effects on efficient utilization of the sensed spectrum.

The main contributions of this study are listed as follows:

- We have generalized the study for realistic signal models which can be applied to any multicarrier CR system.

Until now, simplistic CP-OFDM signal models have been used as the PU and CR signal models for spectrum allocation algorithms [23-26,29-32]. Except for $[27,28]$, CP-OFDM has also been used for the CR. The primary knowledge we assume about the PU waveform is its transmitted power spectral density (PSD) and the receiver selectivity mask; otherwise, there are no limitations regarding the $\mathrm{PU}$ signal model. In our case study, we select the PU waveform either as CP-OFDM following the 802.11-g standard or an FBMC waveform with similar parameterization. Furthermore, a nonlinear transmitter power amplifier model (the so-called Rapp model [33]) is used for the PU system in order to obtain a realistic model for the PU spectrum. To the best of our knowledge, this aspect has not been considered in earlier work. In this way, we are able to quantify the effects of the PU spectral characteristics on the SU capacity. It is seen that the nonlinear power amplifier-induced spectral leakage (regrowth) effect, which is present in any radio communication system, has a significant impact on the SU capacity. As for the SU waveform, we have chosen the FBMC scheme for the case study because it has the sharpest spectrum, reaching the maximum spectral containment among the alternatives. However, generic multicarrier model is included in the overall system model, and the analysis and 
optimization methods are readily applicable for any multicarrier waveform for the CR.

Furthermore, previous studies on $\mathrm{CR}$ resource allocation in [23-25,27-32] consider only flat fading channel models. However, the performance of spectrum sensing and resource allocation is affected significantly when frequency-selective fading channel is assumed. In our study, all the links within/between PU and SU systems associated with spectrum estimation and spectrum utilization are modeled as frequency selective block fading channels.

- Combined spectrum sensing and resource allocation algorithms for cognitive radios.

There has been no previous work addressing the combined spectrum sensing and resource allocation algorithm in the literature. Especially, different types of spectrum sensing algorithms have been applied without considering any particular spectrum utilization techniques to make efficient use of the available spectral holes [1-5]. Similarly, resource allocation algorithms have only been applied without any spectrum sensing information so far [27-32,34,35]. Constant number of available subbands has been considered in the spectral hole. However, the variation of the PUs' power level affects the actual number of available subbands, and this depends critically on the spectral characteristics of the PUs. Hence, spectrum sensing plays a crucial and enabling role for spectrum utilization process. The sensing function identifies the frequency band which is considered for allocation, but it is also needed for detecting possible other PU's starting to operate in the spectral gap during the SU operation. For this purpose, we assume that there are gaps in the CR transmission. In our study, efficient spectrum utilization methods are investigated and applied for maximizing the cognitive radio's throughput based on robust spectrum sensing results. It turns out that the PI algorithm is applicable in our scenario, with all the mentioned generalizations of the system model. The main contribution of this study is evaluating the SU performance with the combination of energy detection-based wideband sensing algorithm and the PI algorithm for spectrum utilization in a realistic cognitive radio scenario.

The rest of this paper is organized as follows. In Section 2, the signal models for the CR and the primary transmission system, along with the mutual interference model between the CR and primary are explained. The problem definition for this study is given in the same section. In Section 3, FFT- and AFB-based wideband spectrum sensing is reviewed considering the spectrum analysis aspects related to the multicarrier techniques. Section 4 develops the algorithms for spectrum allocation. Section 5 gives the numeric and graphic results obtained through simulations. Finally, some concluding remarks are given about the performance of these methods, along with discussion of possible further studies in this area.

\section{Signal models and problem definition}

As shown in Figure 1, the CR system works in the same band of frequencies with PU networks. Hence, there will be some interference between different PUs and CRs. The PU and CR systems are assumed to use the timedivision multiplexing/duplexing (TDMA/TDD) principles, i.e., each system is using a fixed frequency slot for communications between all the stations. While the CR system has the capability to operate in other parts of the ISM band, we focus on the situation where the CR

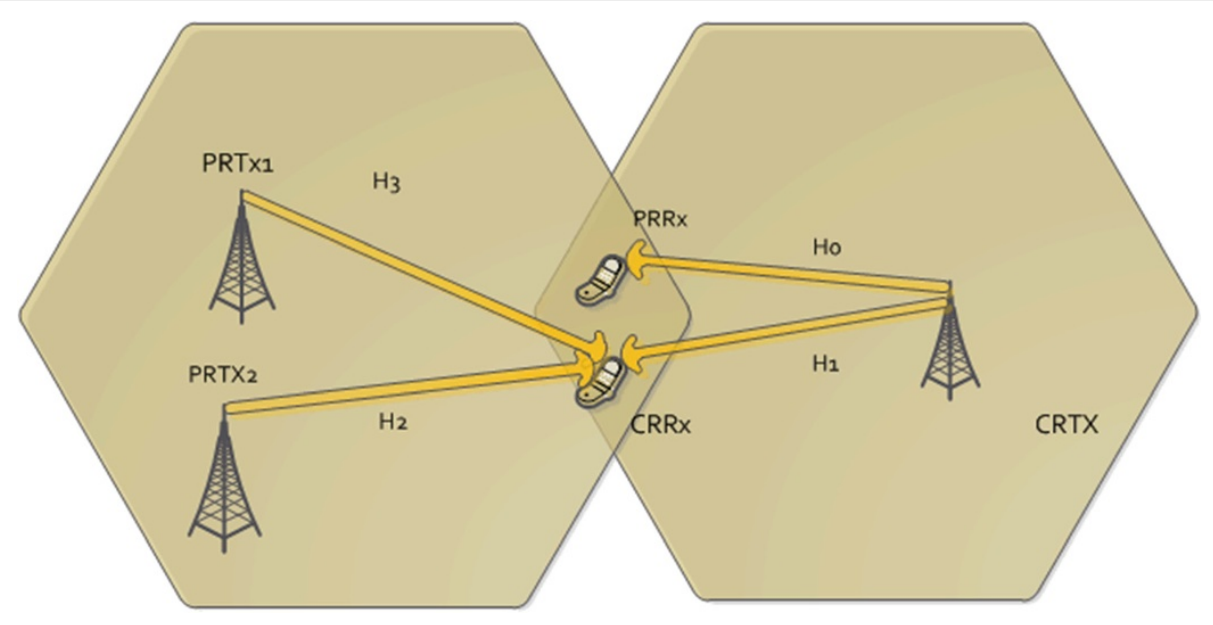

Figure 1 System model for spectrum sharing in CR. 
system has identified a spectrum opportunity in the mentioned frequency slot and is initiating communications in it. The primary purpose of the spectrum sensing function is to detect possible other transmissions or reappearing PUs in the spectrum gap. It is also assumed that the stations of the CR systems have means to exchange control information with each other, e.g., using a cognitive control channel [36].

\subsection{Signal model for PU}

In this study, we focus on a specific spectrum use scenario with two active primary radio systems which are operating in the 2.4-GHz ISM band, using either as 802.11 g-based WLAN waveforms or 802.11 g-like FBMC signals. The WLAN and FBMC spectra considered here use third and eighth channels as illustrated in Figure 2. The signals do not overlap each other, and an $8-\mathrm{MHz}$ spectral hole is available between the two PU spectra. Both active signals are assumed to have the same power level, normalized to $0 \mathrm{~dB}$ in our scenario. This means that the spectrum leakage effects on both edges of the white space are equally critical for the CR system performance.

The Rapp power amplifier (PA) nonlinearity model [33] is considered as seen in Figure 2. Using the complex I/Q baseband model, the amplitude function at the PA output is given as follows:

$$
g_{A}=\frac{\kappa A}{\left(1+\left[\kappa A / A_{0}\right]^{2 p}\right)^{1 / 2 p}}
$$

where $A$ is the input amplitude, $\kappa$ is the small signal gain, $A_{0}$ is the saturated amplitude, and $p$ is the amplitude smoothness factor of the transition from linear to saturated amplitude range. Three cases with respect to the PA nonlinearity are considered in this study. No regrowth is the ideal case, and the Rapp PA nonlinearity with two different back-off values of $15 \mathrm{~dB}$ (modest case) and $5 \mathrm{~dB}$ (worst case) is illustrated in Figure 2. Parameters of the Rapp model have been chosen according to the practical model for PU signals based on [37]. In our study, we use $\kappa=1, p=3$, and $A_{0}=1$ as Rapp model simulation parameters.

The 802.11-g-based WLAN signal specifications allow the spectral regrowth in this scenario to be at the level of about $-20 \mathrm{~dB}$, i.e., close to the worst case model. We investigate how the CR system performance is affected by improved spectral containment of the PU signal through enhanced multicarrier waveform and/or improved power amplifier linearity. These effects for both sensing and utilization functions will be addressed in the study.

\subsection{Signal model for cognitive radio}

In this work, the CR waveform is chosen as FBMC due to its high spectral containment. Offset quadrature amplitude modulation (OQAM) is used for FBMC-based CRs to achieve orthogonality of subcarriers, as discussed in $[18,19,38]$. In Figure 1 , the channels $h_{0}$ and $h_{1}$ are the channels from a cognitive transmitter to a primary receiver and a cognitive receiver, respectively. Channels $h_{2}$ and $h_{3}$ are from two different primary transmitters to the cognitive radio receiver. The channel estimate for $h_{1}$ is made available by usual channel estimation procedure of the CR system. The knowledge about channel $h_{0}$ can be obtained through the channel reciprocity in TDD

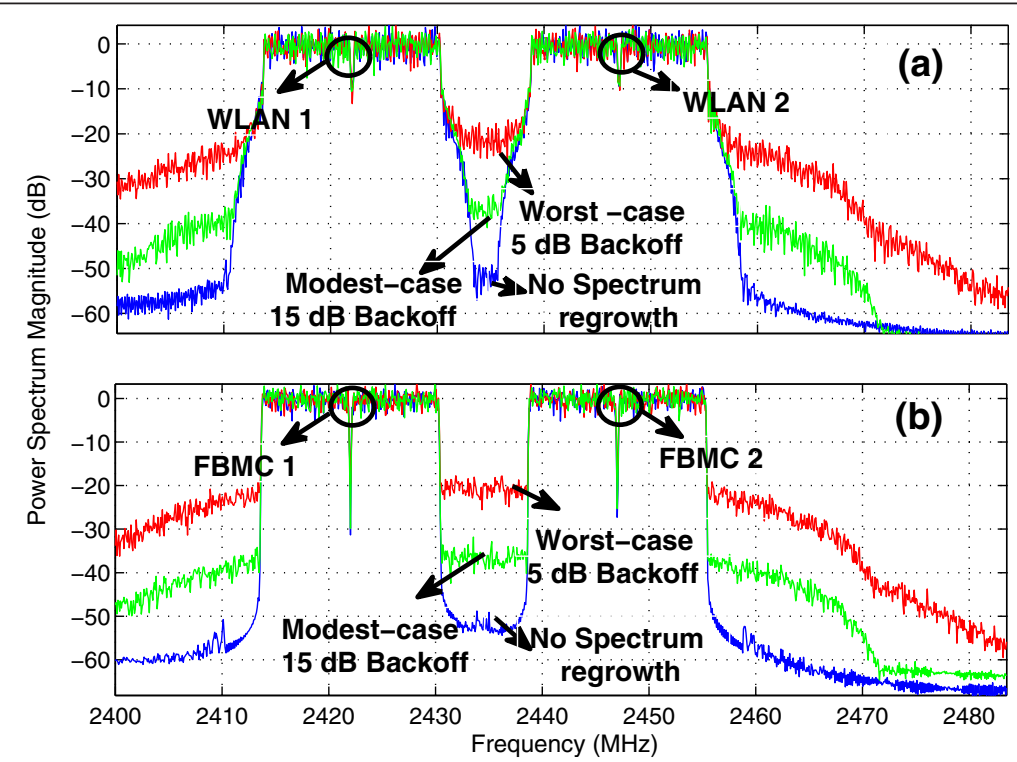

Figure 2 Effects of the Rapp power amplifier model on (a) OFDM- and (b) FBMC-based primary users' spectra. 
operation. Here, the channel amplitude response is sufficient and the phase response is irrelevant. The amplitude responses of channels $h_{2}$ and $h_{3}$ are first obtained through the spectrum sensing function of the CR device and later on, during secondary transmission, through the SINR estimation function of the CR device. The effects of primary spectral dynamics at the edges of the white space play an important role both in spectrum sensing and in spectrum allocation. This dependency is captured by the analytical models and revealed by the simulations to be presented later.

For FBMC/OQAM, a signal model with real valued symbol sequence at twice the QAM symbol rate is applied, instead of complex symbols. The synthesis filter bank (SFB) for transmitter and AFB for receiver is designed with this idea in mind. The FBMC-based transmitted signal can be expressed mathematically as

$$
s(n)=\sum_{k} \sum_{l \in Z} a_{k, l} g\left(n-l \tau_{0}\right) e^{j 2 \pi(k / N) n} e^{j \phi_{k, l}}
$$

where $\{k\}$ is the set of active subbands, $l$ is the symbol index belonging to the set of integers, $g$ is the pulse shape (prototype filter impulse response), and $\phi_{k, l}$ is a phase term. The real symbol values, obtained alternatingly as real and imaginary parts of complex QAM symbols, are denoted as $a_{k, l}$ and $\tau_{0}$, respectively, is the corresponding half-symbol duration. Both the real and imaginary parts of the QAM sequence have zero mean and equal variances $\sigma_{r}^{2}=\sigma_{I}^{2}=\sigma_{s}^{2} / 2$. The PSD of the FBMC based CR waveform can be written as

$$
\phi_{\mathrm{FBMC}}(f)=\frac{\sigma_{r}^{2}}{\tau_{0}} \sum_{k}\left|G\left(f-\frac{k}{N}\right)\right|^{2}
$$

where $G$ is the frequency response of the prototype filter with impulse response $g(n)$ with $n=0,1 \ldots, L-1$. Here, $L=K N$ is the prototype filter length and $K$ is the overlapping factor (length of each polyphase component) while $N$ is the number of subbands. The prototype coefficients have even symmetry around the $(K N / 2)$ th coefficient, and the first coefficient is zero [13]. In our study, the prototype filter of the FBMCbased $\mathrm{CR}$ is designed according to the PHYDYAS model [38], with $K=4$. Then its frequency response can then be expressed as

$$
|G(f)|=g[L / 2]+2 \sum_{r=1}^{L / 2-1} g[L / 2-r] \cos (2 \pi f r)
$$

Also, the nonlinear PA model can be straightforwardly included in the $\mathrm{CR}$ signal model and the interference models developed below. However, in the numerical studies of this paper, we consider an ideal FBMC waveform for the CR as the focus is on the SU capacity and its dependency on the PU waveform characteristics. Generally, good spectral containment is regarded as one of the key requirements for the CR transmitter. Detailed evaluation of the performance-complexity tradeoffs for the $\mathrm{CR}$ implementation, including the PA linearity requirements, is a rather complicated issue and is left as a topic for future studies.

\subsection{Definition of the interference problem}

According to the above scenario, the CR system coexists with the primary transmission system in the same geographical location. The $\mathrm{CR}$ transmitter causes some interference to the primary transmission system, and similarly, the secondary transmission between two active PU spectra is exposed to some interference due to the PUs. The secondary transmission system uses multicarrier transmission technique. There are $N_{\text {gap }}$ subcarriers in the sensed spectrum hole and the subcarrier spacing is $\Delta f$. Since the transmitter and receiver are assumed to be static or slowly moving, the effect of inter-carrier interference (ICI) between subcarriers can be ignored. The primary and secondary transmission systems occupy contiguous frequency slots. The interference that the CR produces to each of the primaries is required to be is less than the maximum interference that can be tolerated by the primary, Ith. The spectral distance $d_{\mathrm{PU}}$ of a $\mathrm{PU}$ is defined as the frequency separation from the DC subcarrier of the CR to the center frequency of the PU (positive for a PU above the upper edge of the gap, negative for a PU below the gap). The interference to the primary transmission due to the $k$ th $C R$ subcarrier depends on the CR subcarrier powers $P_{k}$ and $d_{\mathrm{PU}}$ [20]. Fixing the origin of the frequency axis at the DC subcarrier of the $\mathrm{CR}$, the interference is given by the equation

$$
I_{k}\left(P_{k}\right)=\int_{k \Delta f-B / 2}^{k \Delta f+B / 2}\left|H_{0}(f)\right|^{2} P_{k} \Phi(f-k \Delta f) \Psi\left(f-d_{\mathrm{PU}}\right) d f=P_{k} \Omega_{k}
$$

Here, $H_{0}(f)$ is the channel frequency response between the CR transmitter and a primary receiver. $\Phi(f)$ represents the subcarrier power spectral density of the underlying multicarrier technique employed by the CR. $\Psi(f)$ denotes the PU sensitivity mask characterizing the effects of the PU receiver filtering. $B$ denotes the CR subcarrier bandwidth which is considered significant for the interference estimation. Finally, $\Omega_{k}$ represents the combined interference factor for the $k$ th $C R$ subcarrier. 
The signal-to-interference-plus-noise ratio due to interference introduced by primary signal to the $k$ th subcarrier at the receiving $\mathrm{CR}$ is given by

$$
\begin{aligned}
\operatorname{SINR}_{k} & =\frac{P_{k}\left|H_{1, k}\right|^{2}}{\sigma_{w}^{2}+\int_{k \Delta f-B / 2}^{k \Delta f+B / 2}\left|H_{2}(f)\right|^{2} \Phi(f-k \Delta f) \psi_{\mathrm{PA}}\left(f-d_{\mathrm{PU}}\right) d f} \\
& =\frac{P_{k}}{\sigma_{w}^{2}+J_{k}}
\end{aligned}
$$

where $H_{2}(f)$ is the channel frequency response between the primary transmitter and $\mathrm{CR}$ receiver. $H_{1, k}$ is the channel gain between the CR transmitter and the CR receiver at the frequency of $k$ th subcarrier. This channel can be assumed to be flat-fading at the subcarrier level. $\Psi_{\mathrm{PA}}(f)$ is the power spectral density as seen at the output of the PU's transmitter antenna. This depends on the PU transmission power and its subcarrier spectrum, as well as on the spectral regrowth due to the PU power amplifier. $\Phi(f)$ is the CR receiver sensitivity mask characterizing the CR receiver subband filtering effects. $\sigma_{w}^{2}$ is the variance of the additive white Gaussian noise.

The power amplifier effects of the secondary transmission are not considered in our numerical study as they play no role in the spectrum sensing part and the effect of PA-related spectrum leakage on the interference to the PUs is expected to be relatively small. For this reason, the same $\Phi(f)$ function can be used in (5) for the CR subcarrier spectrum and in (6) for the CR receiver sensitivity mask. However, the developed generic signal model allows to include also the CR transmitter PA effects by using different CR-related spectral functions in (5) and (6).

The interference models of (5) and (6) assume certain knowledge about PU characteristics and the channels between PUs and CRs. Regarding the interference from an active PU transmitter to a CR receiver in (6), the joint effect of transmitter power spectrum and the transmission channel can be estimated by the receiving CR station by utilizing the spectrum sensing function. This information can be communicated through the control channel to the transmitting CR station for optimizing the spectrum utilization. Regarding the channel from the $\mathrm{CR}$ transmitter to PU receiver, the knowledge would be available in a TDMA/TDD-based PU system (like a WLAN) based on channel reciprocity, if the PU transmission power is known. Of course, for a PU station which is in idle mode over long periods, such information is not available.

\section{Filter bank energy detector-based spectrum sensing algorithms}

Energy detector, which is also known as radiometer, is the most common method of spectrum sensing due to its low computational and implementation complexity [2-5]. Furthermore, it is more generic compared to most of the other methods as it does not need any information about the PU waveform. Subband-based energy detection, using FFT or AFB for spectrum analysis, is in the focus of this study. Basically, the energy of the received signal is compared with a threshold value which is calculated according to noise variance and desired false alarm probability in detecting spectral holes.

A block diagram of alternative FFT- and AFB-based spectrum sensing algorithms is shown in Figure 3. The subband sampling rate is equal to the ADC sampling rate divided by the number of FFT/AFB frequency bins. With subband-wise spectrum sensing method, the subband signals can be expressed as [3]

$$
Y(m, k)=\left\{\begin{array}{ll}
W(m, k) & \mathcal{H}_{0} \\
S(m, k) H_{k}+W(m, k) & \mathcal{H}_{1}
\end{array}\right\}
$$

where $S(m, k)$ is the transmitted WLAN or FBMC based PU signals as seen in subband $k$ during the $m$ th symbol interval with zero mean and variance $\sigma_{\mathrm{PU}}^{2}$. When there are no PU signals (hypothesis $\mathcal{H}_{0}$ ), the noise samples $W$ $(m, k)$ are modeled as AWGN with zero-mean and variance $\sigma_{w}^{2}$. When a PU signal is present (hypothesis $\mathcal{H}_{1}$ ), the WLAN- and FBMC-based PU signals can also be modeled as zero-mean Gaussian distribution with variance $\sigma_{\mathrm{PU}, k}^{2}+\sigma_{w}^{2}$.

Time and frequency averaging techniques can be applied to obtain more reliable decision statistic [3]. The decision statistics at different frequencies can be obtained with this idea as follows in [39]:

$$
\tilde{T}(m, k)=\frac{1}{L_{t} L_{f}} \sum_{l=k-\left\lfloor L_{f} / 2\right\rfloor}^{k+\left\lceil L_{f} / 2\right\rceil-1} \sum_{u=m-L_{t}+1}^{m}|Y(u, l)|^{2}
$$

In this formula, $L_{f}$ and $L_{t}$ are the window lengths in frequency and time domain averaging, respectively. The output of $\tilde{T}(m, k)$ is passed to a decision device to determine the possible occupancy of the corresponding frequency band at the corresponding time interval. The window length in frequency direction is selected based on the expected minimum bandwidth of the PU signal or spectrum hole, and then the required time domain window length can be calculated from the target false

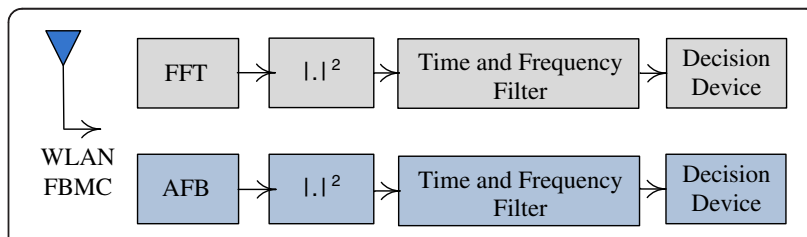

Figure 3 Block diagram of energy detector with AFB- and FFT-based spectrum analysis. 
alarm and missed detection probabilities. The basic approach would be to calculate (8) for a nonoverlapping set of windows. However, using a sliding window in frequency and/or time direction can also be done with relatively small addition to complexity. Time-domain sliding window helps to detect rapidly re-appearing PU $[4,5]$ whereas sliding window in the frequency direction helps to locate spectrum gaps with unknown center frequencies. Due to the Gaussian distribution of $Y(m, k)$, the probability distribution function (PDF) of $\tilde{T}(m, k)$ becomes approximately Gaussian under $\mathcal{H}_{0}$ and $\mathcal{H}_{1}$. [3].

Using Gaussian approximation, it is straightforward to model the effect of PU transmitter's spectral leakage on the actual false alarm probability $\tilde{P}_{\text {FA }}$ as

$$
\tilde{P}_{\mathrm{FA}}(k)=Q\left(\frac{\lambda-\left(\sigma_{w}^{2}+I_{\mathrm{adj}}(k)\right)}{\sqrt{\frac{1}{L_{t} L_{f}}}\left(\sigma_{w}^{2}+I_{\mathrm{adj}}(k)\right)}\right)
$$

Here,

$$
I_{\text {adj }}(k)=\int_{f_{1}}^{f_{2}}\left|H_{2}(f)\right|^{2} \psi_{\mathrm{PA}}(f) d f
$$

is the leakage power from the adjacent PU transmitter to the sensing frequency band between frequencies $f_{1}$ and $f_{2} . H_{2}(f)$ is the channel frequency response from a primary transmitter the CR receiver. In (9), $\lambda$ is the decision threshold value which is calculated using a wellknown analytical model from the noise variance estimate and target false alarm probability $P_{\mathrm{FA}}$.

The detection probability $P_{D}$ and threshold value $\lambda$ can also be expressed as follows:

$$
\begin{aligned}
& P_{D}(k)=Q\left(\frac{\lambda-\left(\left(\sigma_{w}^{2}+I_{\mathrm{adj}}(k)\right)+\sigma_{\mathrm{PU}, k}^{2}\right)}{\sqrt{\frac{1}{L_{t} L_{f}}}\left(\left(\sigma_{w}^{2}+I_{\mathrm{adj}}(k)\right)+\sigma_{\mathrm{PU}, k}^{2}\right)}\right) \\
& \lambda=Q^{-1}\left(P_{\mathrm{FA}}(k)\right) \sqrt{\frac{1}{L_{t} L_{f}}}\left(\sigma_{w}^{2}+I_{\mathrm{adj}}(k)\right)+\left(\sigma_{w}^{2}+I_{\mathrm{adj}}(k)\right)
\end{aligned}
$$

In principle, if there is a reliable estimate of the PU transmission power and reliable knowledge about its spectrum shape, then the above analysis could be used for improving the spectrum sensing at the frequencies affected by the spectrum leakage. However, this would be very challenging in practice due to the unpredictability of the PA characteristics, and the above model is used only for the purpose of performance analysis.

For different PU SNR values, different number of empty subbands, $N_{\text {gap }}$, will be detected due to the PU spectral leakage effects and statistical nature of the spectrum sensing process. The expression (9) can be used for evaluating the false alarm probability for different sensing bandwidths in different parts of the spectrum gap.

The spectrum sensing function identifies groups of $L_{f}$ subbands which are deemed to be available for secondary transmissions. In the following stage, the spectrum utilization function is employed to perform power and bit allocation to those subcarriers.

\section{Spectrum utilization}

After nonactive spectrum has been identified, spectrum utilization becomes an important consideration, when considering the overall efficiency of the CR system. The number of available nonactive subbands is the output of the sensing algorithm, along with information about the nonactive band edges.

In the multicarrier case, the rate at which transmission can take place is given by Shannon's capacity

$$
\begin{aligned}
& R_{\mathrm{CR}}=\sum_{k=1}^{N_{\text {gap }}} \Delta f \log _{2}\left(1+\frac{P_{k}}{\sigma_{k}^{2}}\right) \\
& \sigma_{k}^{2}=\sigma_{w}^{2}+\sum_{i=1}^{N_{\mathrm{PU}}} J_{k, i}
\end{aligned}
$$

where $J_{k, i}$ is the effective interference power contributed by $i$ th primary to the $k$ th CR subcarrier as given by (6). $N_{\text {PU }}$ is the number of PU's contributing to the interference at the receiving $\mathrm{CR}$ station. In our case study, $N_{\mathrm{PU}}=$ 2, i.e., there is one PU adjacent to the lower and upper edges of the white space. The model could be simplified by assuming that these PUs affect only the lower and upper half of the subcarriers, respectively. $P_{k}$ is the transmit power used by the CR for subcarrier $k$. It is assumed that the channel changes slowly so that the channel gains, and consequently $J_{k, i}$, will be approximately the same during each transmission frame. Further, there is no ICI in the $\mathrm{CR}$ reception due to low mobility. The main objective here is to maximize the capacity as given in (13).

The block diagram of spectrum utilization is shown in Figure 4. As seen in this block diagram, knowledge which comes from sensing part is passed to spectrum utilization part to obtain better capacity.

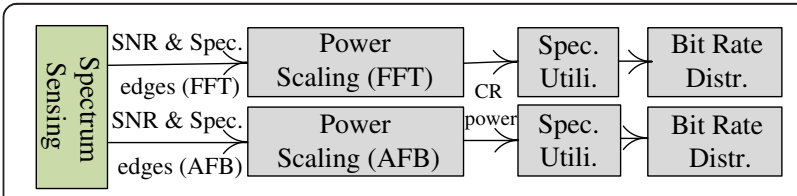

Figure 4 Block diagram of spectrum utilization with alternative AFB- and FFT-based spectrum analysis schemes. 
The optimization problem can be formulated as follows [27]:

$$
R_{\mathrm{CR}}=\max _{\left\{P_{k}\right\}} \sum_{k=1}^{N_{\text {gap }}} \Delta f \log _{2}\left(1+\frac{P_{k}}{\sigma_{k}^{2}}\right)
$$

Subject to

$$
\begin{aligned}
& \sum_{k=1}^{N_{\text {gap }}} P_{k} \leq P_{T} \\
& \sum_{k=1}^{N_{\text {gap }}} P_{k} \Omega_{k} \leq I_{\text {th }} \\
& P_{k} \geq 0, \forall k \in\left\{1,2, \ldots, N_{\text {gap }}\right\}
\end{aligned}
$$

This is a convex optimization problem, and the Lagrangian can be written as

$$
\begin{aligned}
& G_{\text {snr }}=\sum_{k=1}^{N_{\text {gap }}} \Delta f \log _{2}\left(1+\frac{P_{k}}{\sigma_{k}^{2}}\right) \\
& -\lambda_{0} \sum_{k=1}^{N_{\text {gap }}}\left(P_{k}-P_{T}\right)-\lambda_{1}\left(P_{k} \Omega_{k}-I_{\text {th }}\right)+\lambda_{2} \sum_{k=1}^{N_{\text {gap }}} P_{k}
\end{aligned}
$$

The Karush-Kuhn-Tucker (KKT) conditions [27] can be written as

$$
\begin{aligned}
& P_{k} \geq 0, \forall k \in\left\{1,2, \ldots, N_{\text {gap }}\right\}, \\
& \lambda_{j} \geq 0, \forall j \in\{0,1,2\} \\
& \sum_{k=1}^{N_{\text {gap }}}\left(P_{k}-P_{T}\right) \geq 0 \\
& \lambda_{1}\left(P_{k} \Omega_{k}-I_{\text {th }}\right) \geq 0 \\
& \lambda_{2} \sum_{k=1}^{N_{\text {gap }}} P_{k} \geq 0
\end{aligned}
$$

The optimal solution to the problem above as given in [27] is as follows:

$$
P_{k}=\left[\frac{1}{\lambda_{0} \Omega_{k}+\lambda_{1}}-\frac{\sigma_{k}^{2}}{\left|h_{k}\right|}\right]^{+}
$$

where $[y]^{+}=\max (0, y)$. The optimal solution has high computationally complexity; hence, a lower complexity algorithm called the PI algorithm which divides the problem into stages has been developed [27]. First, the interference constraint is ignored, keeping only the total power constraint and this leads to the classical water filling solution

$$
P_{k}{ }^{\prime}=\left[\gamma-\frac{\sigma_{k}^{2}}{\left|h_{k}\right|}\right]^{+}
$$

where $\gamma$ is the water filling level. When the total power is ignored the solution [27] becomes

$$
P_{k}{ }^{\prime}=\left[\frac{1}{\lambda_{0}^{\prime} \Omega_{k}}-\frac{\sigma_{k}^{2}}{\left|h_{k}\right|}\right]^{+}
$$

The value of $\lambda_{0}^{\prime}$ can be obtained by substituting (27) into the constraint $\sum_{k=1}^{N_{\text {gap }}} P_{k}^{\prime} \Omega_{k}=I_{\text {th }}$ to get

$$
\lambda_{0}^{\prime}=\frac{\left|N_{\text {gap }, l}\right|}{I_{\text {th }}^{N_{\text {gap }}}+\left(\sum_{i} \Omega_{k} \sigma_{k}^{2} /\left|h_{k}\right|^{2}\right)}
$$

The above solution is optimal only when the total power is greater than or equal to the power under the interference constraint. Mostly, in practice, this condition is not true and this is the motivation for the PI algorithm. Detailed discussion and its comparison to various other algorithms for spectrum utilization are available in [27].

In this study, the PI algorithm is found to be directly applicable in case of the developed greatly enhanced system model for the secondary usage scenario. PI algorithm has four stages: maximum power determination, power constraint, power budget distribution, and power level re-adjustment [27].

\section{Simulation results}

In our test scenario, the CR's spectrum sensing function has identified a potential spectral gap between two relatively strong PUs, as illustrated in Figure 2. We should also consider the possibility that there is another, relatively weak PU signal, using one of the WLAN channels $4 \ldots 7$, and fully or partly occupying the gap between channels 3 and 8 . Thus, one purpose of spectrum sensing is to secure that there are no other PUs active in the considered gap. We assume that there are no additional signals within the spectral gap, but the spectrum sensing makes anyway false alarms. Especially close to the edges of the gap, the spectrum leakage from the PUs raises the false alarm probability. This effect depends on the power level (SNR) of the PUs. In our case study, the spectrum sensing and $\mathrm{CR}$ transmissions use a smaller subband spacing of $81.5 \mathrm{kHz}$, instead of the $325-\mathrm{kHz}$ subcarrier spacing of WLANs, in order to reduce the effects of frequency selective channels. Targeting at $-5 \mathrm{~dB}$ SNR in spectrum sensing, false alarm probability of 0.1 , and detection probability of $90 \%$, the required sample complexity is around 250 complex samples. The time and frequency averaging lengths are chosen as 50 and 5, respectively. The spectral hole starts from the side lobes of WLAN 1 signal and ends at the side lobes of WLAN 2 spectrum. The available number of subbands/bandwidth 
of the spectrum is obtained after subband-based energy detection, using FFT or AFB for spectrum analysis. Then, the initial SINR estimation and spectrum allocation is done based on the sensing results. Later on, the SINR estimates are updated during SU system operation to track the changing radio environment under frequency-selective fading channel conditions. It is assumed that the spectrum sensing is done in regular intervals during gaps in the $\mathrm{CR}$ transmission and this helps in detecting reappearing $\mathrm{PU}$ signals in the spectral gap.

It should be noticed that in the considered scenario, there is no way for the CR system to determine the useful received power level at the PU receiver. Therefore, we choose the interference threshold to be $6 \mathrm{~dB}$ below the thermal noise level, in order not to introduce significant performance loss in case the primary receiver is operating close to the sensitivity level (i.e., minimum received power level expected to be detectable). To determine the threshold value, we assume a simplified scenario, where the path losses of channels $h_{0}$ and $h_{1}$ are normalized to 1 , i.e., the average power gains of channels $h_{0}$ and $h_{1}$, denoted as $G_{0}$ and $G_{1}$, are equal to one. Further, we assume that the average SNR of the CR receiver is $10 \mathrm{~dB}$. Then, the interference threshold is $-16 \mathrm{~dB}$ in reference to the total CR transmission power $P_{T}$, or $I_{\text {th }}=$ $P_{T} / 40$. More generally, relaxing the normalization of $h_{0}$ and $h_{1}$, this can be expressed as $I_{\text {th }}=G_{1} P_{T} /\left(40 G_{0}\right)$.

The bandwidth of the detected spectral hole is shown in Figure 5 as a function of the average PU SNR at the
CR RX. The spectral leakage due to primary users' PA nonlinearity is affecting significantly on the width of the detected hole. In this respect, we consider three different cases, as explained in Subsection 2.1: ideal PA, modest PA nonlinearity with $15 \mathrm{~dB}$ back-off, and worst case nonlinearity with $5 \mathrm{~dB}$ back-off. All PU and CR channels $h_{0}, h_{1}, h_{2}$, and $h_{3}$ use frequency-selective channel models with 90 ns delay spread and 16 taps [40]. We consider the combinations of two PU waveforms, CP-OFDM- and FBMC-based WLANs, as well as two spectrum sensing techniques, based on FFT or AFB. The CR waveform is always FBMC.

From Figure 5, it can be easily seen that AFB-based spectrum sensing is able to detect the unoccupied spectrum close to strong primary users much better than FFT-based sensing. FBMC-based transmission results in much better spectral containment, which can be effectively utilized by AFB-based sensing. However, even with relatively modest power amplifier nonlinearity, this benefit of FBMC waveform is compromised.

In Figure 6, the actual false alarm probability within the spectral hole is shown as a function of the active PU's SNR for different levels of spectral regrowth. The results indicate the probability of the 5 subband groups to be detected to be occupied.

The efficiency of the utilization of the $8-\mathrm{MHz}$ white space by $\mathrm{SU}$ in between two active PUs is shown in Figure 7 versus the PU SNR. In this figure, perfect CSI is considered for the CR channel, both for CR channel $h_{1}$

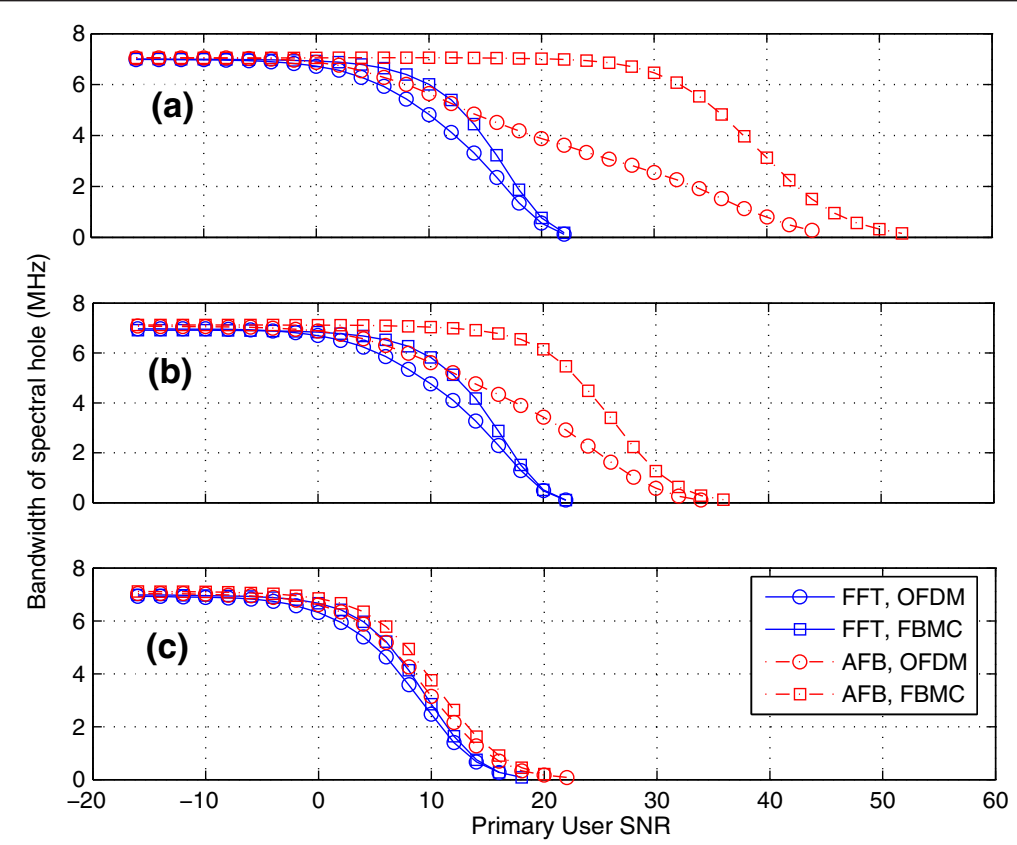

Figure 5 Average bandwidth of the detected spectral hole with target $\boldsymbol{P}_{\mathrm{FA}} \mathbf{= 0 . 1}$. Using sample complexity of 250 samples under frequencyselective channel model for (a) ideal model, (b) Rapp PA with $15 \mathrm{~dB}$ back-off as the modest case, (c) Rapp PA with 5 dB back-off as the worst case. Different combinations of FFT/AFB-based sensing and OFDM/FBMC based PU waveforms. 

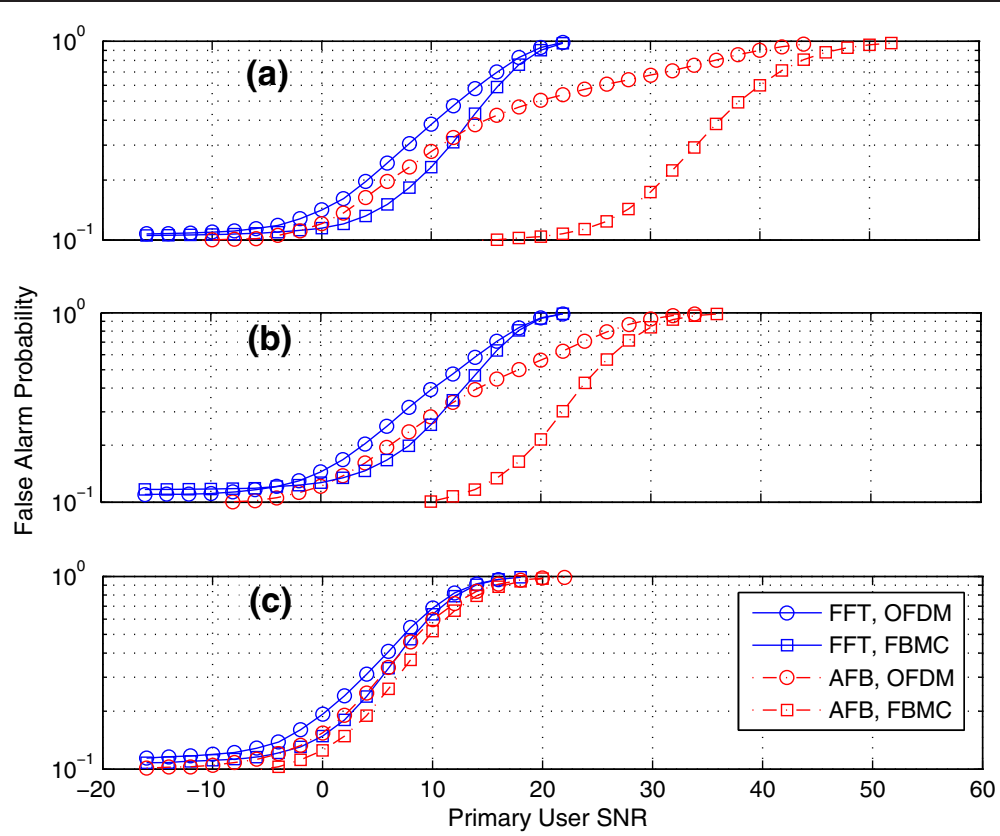

Figure 6 Actual false alarm probability for target $P_{\mathrm{FA}}=\mathbf{0 . 1}$. Time record length of 50 and sensing bandwidth of 5 subbands under frequency selective channel model for (a) ideal model, (b) Rapp PA with $15 \mathrm{~dB}$ backoff as modest case, (c) Rapp PA with $5 \mathrm{~dB}$ backoff as worst case. Different combinations of FFT/AFB based sensing and OFDM/FBMC based PU waveforms.

equalization and in the PI algorithm for resource allocation. Perfect knowledge of the amplitude response of channel $h_{0}$ is also assumed, while channels $h_{2}$ and $h_{3}$ are known from spectrum sensing results. The subbandwise noise + interference estimates are obtained using time filtering length of 50 . According to FFT- or AFBbased spectrum sensing results, a number of subbands are left empty in the spectrum utilization phase. The power of these occupied subchannels is reallocated to the other subbands that can be used by the CR. The
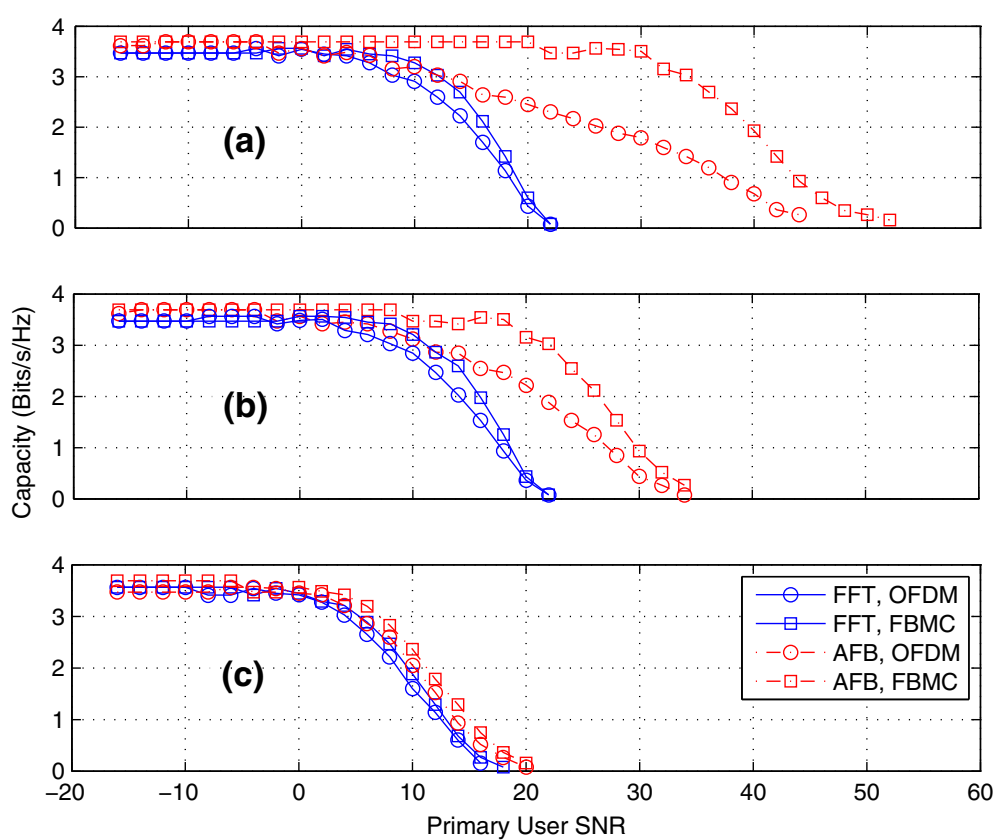

Figure 7 Capacity of a CR in a spectral gap between two PUs versus PU SNR. PI algorithm used for power allocation (a) Ideal model, (b) Rapp PA with $15 \mathrm{~dB}$ backoff as the modest case, (c) Rapp PA with $5 \mathrm{~dB}$ backoff as the worst case. Different combinations of FFT/AFB-based sensing and OFDM/FBMC-based PU waveforms. 
power allocation is done utilizing the PI algorithm, and the resulting capacity, in terms of bits $/ \mathrm{s} / \mathrm{Hz}$, is shown in Figure 7. The limitations of FFT-based spectrum sensing can again be clearly seen from the results, whereas AFBbased sensing is able to identify gaps between relatively strong PU signals. Regarding the transmission waveform, FBMC has clear benefit due to better spectral containment, if the effects of power amplifier nonlinearities can be kept at a modest level. As expected, the capacity is proportional to the available bandwidth in the gap, i.e., there is a clear connection between the results of Figures 5 and 6 . The resource allocation algorithm (i) optimizes the performance with frequency selective channels in the presence of spectral leakage from the strong PUs and (ii) secures that the interference leakage from the CR transmission to the primaries is at an acceptable level.

\section{Conclusions}

We have studied the effects of combined spectrum sensing and spectrum utilization for FBMC-based cognitive radios with realistic signal model under frequencyselective fading channel conditions. Firstly, the performance of energy detection-based spectrum sensing technique was analyzed using both the FFT and filter bank-based spectrum analysis methods for both WLAN and FBMC signal models. Then, the utilization of dynamically identified spectral holes with spectrum allocation algorithms, subject to power and interference constraints, was investigated. Through this study, the effect of PU waveform's spectral containment on the CR transmission capacity was revealed. Here, we considered the choice between OFDM and FBMC primaries, together with the effect of spectral regrowth due to power amplifier nonlinearity.

In terms of the spectrum sensing performance, AFB has clear benefits due to much better spectral containment of the subbands. One important benefit of FBMC as a transmission technique in CR systems is that it can utilize narrow spectral gaps in an effective and flexible way, even in the presence of strong primaries at the adjacent spectral slots. This is due to the excellent spectral containment properties of the FBMC system. Additionally, an FBMC receiver can use the AFB for high-performance spectrum sensing with no additional complexity.

The utilization of the sensed spectrum can be optimized by using proper spectrum allocation algorithms. The PI algorithm has relatively low complexity, and it improves the capacity of the CR system as compared to the simple water filling-based spectrum allocation. One of the main observations of this work was that the PI algorithm can be directly utilized with the developed highly enhanced and realistic CR system model. The system model accommodates frequency-selective channel models for all the associated transmission links between PUs and SUs, as well as arbitrary transmitted power spectra and receiver frequency responses. Because of the above features, a FBMC-based CR system achieves higher capacity in comparison with traditional WLANbased system. This increase in capacity can be attributed to the efficient use of the available spectrum and very small interference introduced to the primary transmissions at adjacent frequencies.

One of the important aims of this study was to understand the interdependence of the spectrum sensing and the spectrum utilization parts. It can be seen that increased false alarm probability has a direct effect on the available spectrum, and hence, it heavily influences the spectrum utilization. The PU power amplifier nonlinearity influences the sensed secondary spectrum introducing false alarms, hence lowering the CR system's spectrum utilization. It was demonstrated that, with heavy power amplifier nonlinearity, the FBMC-based primary is no better than the OFDM primary in what comes to the available capacity for secondary usage in the nearby frequencies.

In the numerical studies of this paper, we considered an ideal FBMC waveform for the CR, without considering the PA nonlinearity effects, since the focus is on the SU capacity and it dependency on the PU signal characteristics. Generally, good spectral containment is regarded as one of the key requirements for the CR transmitter. Also, the nonlinear PA model can be straightforwardly included in the developed interference models. Detailed evaluation of the performance-complexity tradeoffs for the CR implementation, including the PA linearity requirements, is a rather complicated issue and is left as a topic for future studies.

\section{Competing interests}

The authors declare that they have no competing interests.

\section{Acknowledgements}

This work was partially supported by Tekniikan Edistämissäätiö (TES), GETA Graduate School, the European Commission under Project EMPhAtiC (FP7ICT-2012-09-318362), COST Action IC0902, Tekes (the Finnish Funding Agency for Technology and Innovation) under the project ENCOR in the Trial Program.

\section{Author details}

${ }^{1}$ Department of Electronics and Communications Engineering, Tampere University of Technology, P.O. Box 692, Tampere 33101, Finland. ${ }^{2}$ Centre Tecnològic de Telecomunicacions de Catalunya (CTTC), Parc Mediterrani de la Tecnología (PMT), Avenida Carl Friedrich Gauss 7, 08860 Barcelona, Spain. ${ }^{3}$ Signal, Communication \& Embedded Electronics Research Group, Ecole Supérieur D'Électricité -SUPELEC University, F-35576 Cesson-Sévigné Cedex, Gif-sur-Yvette C.S. 47601, France.

Received: 10 December 2013 Accepted: 15 April 2014 Published: 12 May 2014

\section{References}

1. J Mitola, JGQ Maguire, Cognitive radio: making software radios more personal. IEEE Pers Commun Mag 6(4), 13-18 (1999) 
2. $\quad Y$ Zeng, $Y C$ Liang, AT Hoang, $R$ Zhang, A review on spectrum sensing for cognitive radio: challenges and solutions. EURASIP J Adv Signal Process 2010, 1-15 (2010)

3. T Yucek, H Arslan, A survey of spectrum sensing algorithms for cognitive radio applications. IEEE Commun Surv Tutorials 11(1), 116-130 (2009)

4. S. Dikmese, M. Renfors, H. Dincer, FFT and filter bank based spectrum sensing for WLAN signals, in Proc (European Conference on Circuit Theory and Design (ECCTD'11) (Linkoping, Sweden, 2011)

5. S Dikmese, M Renfors, Optimized FFT and filter bank based spectrum sensing for bluetooth signal, in Proc (Wireless Communications and Networking Conference (WCNC'12) (France, Paris, 2012)

6. AJ Goldsmith, PP Varaiya, Capacity of fading channels with channel side information. IEEE Trans Inf Theory 43(6), 1986-1992 (1997)

7. E Biglieri, J Proakis, S Shamai, Fading channels: information-theoretic and communications aspects. IEEE Trans Inf Theory 44(6), (1998)

8. M Gastpar, On capacity under received-signal constraints, in Proc (42nd Annual Allerton Conference Communication (Control Comput, Monticello, USA, 2004)

9. A Ghasemi, E Sousa, Fundamental limits of spectrum-sharing in fading environments. IEEE Trans Wirel Commun 6(2), 649-658 (2007)

10. L Musavian, S Aissa, Ergodic and outage capacities of spectrum-sharing systems in fading channels, in Proc (IEEE Global Telecommunications Conference (GLOBECOM'07), Washington (DC, USA, 2007)

11. X Kang, YC Liang, A Nallanathan, HK Garg, R Zhang, Optimal power allocation for fading channels in cognitive radio networks: ergodic capacity and outage capacity. IEEE Trans Wirel Commun 8(2), 940-950 (2009)

12. A Sahin, H Arslan, Edge windowing for OFDM based systems. Commun Lett IEEE 15(11), 1208-1211 (2011)

13. S Brandes, I Cosovic, M Schnell, Sidelobe suppression in OFDM systems by insertion of cancellation carriers,' in Proc (Vehicular Technology Conference, (VTC'05-Fall) (Dallas, USA, 2005)

14. A Loulou, M Renfors, 'Effective schemes for OFDM sidelobe control in fragmented spectrum use, in Proc (IEEE 24th International Symposium on Personal, Indoor and Mobile Radio Communications (PIMR'13) (United Kingdom, London, 2013)

15. BF Boroujeny, R Kempter, Multicarrier communication techniques for spectrum sensing and communication in cognitive radios. IEEE Commun Mag (Spec Issue Cogn Radios Dynamic Spectr Access) 48(4), 80-85 (2008)

16. A Amini, R Kempter, L Lin, B Farhang-Boroujeny, Filter bank multitone: a candidate for physical layer of cognitive radio, in Proc (Software Defined Radio Technical Conference and Product Exhibition (SDR '05) (Orange County, USA, 2005)

17. A Amini, R Kempter, B Farhang-Boroujeny, A comparison of alternative filterbank multicarrier methods in cognitive radios, in Proc (Software Defined Radio Technical Conference and Product Exhibition (SDR '06) (Orlando, USA, 2006)

18. M Bellanger, $T$ Ihalainen, M Renfors, Filter bank based cognitive radio physical layer, in Proc (Future Network \& Mobile Summit, Santander, Spain, 2009)

19. T Ihalainen, A Viholainen, TH Stitz, M Renfors, Spectrum monitoring scheme for filter bank based cognitive radios, in Proc (Future Network \& Mobile Summit, Florence, Italy, 2010)

20. S Srinivasan, S Dikmese, M Renfors, Spectrum sensing and spectrum utilization model for OFDM and FBMC based cognitive radios, in Proc (Signal Processing Advances in Wireless Communications (SPAWC'12) (Cesme, Turkey, 2012)

21. V Ringset, H Rustad, F Schaich, J Vandermot, M Najar, Performance of a filterbank multicarrier (FBMC) physical layer in the WiMAX context, in Proc (Future Network \& Mobile Summit, Florence, Italy, 2010)

22. S Dikmese, S Srinivasan, M Renfors, FFT and filter bank based spectrum sensing and spectrum utilization for cognitive radios, in Proc (International Symposium on Communications, Control, and Signal Processing (ISCCSP'12) (Rome, Italy, 2012)

23. G Bansal, MJ Hossain, VK Bhargava, Adaptive power loading for OFDM-based cognitive radio systems, in Proc (IEEE International Conference on Communications (ICC '07) (Glasgow, UK, 2007)

24. G Bansal, MJ Hossain, VK Bhargava, Optimal and suboptimal power allocation schemes for OFDM-based cognitive radio systems. IEEE Trans Wirel Commun 7(11), 4710-4718 (2008)

25. T Qin, C Leung, Fair adaptive resource allocation for multiuser OFDM cognitive radio systems, in Proc (2nd International Conference on Communications and Networking in China (ChinaCom '07) (Shanghai, China, 2007)

26. Y Zhang, Resource allocation for OFDM-based cognitive radio systems (University of British Columbia, Vancouver, Canada, 2008). Dec, Ph.D. dissertation
27. M Shaat, F Bader, Computationally efficient power allocation algorithm in multicarrier-based cognitive radio networks: OFDM and FBMC systems. EURASIP J Adv Signal Process 13, (2010). doi:Article ID 528378

28. M Shaat, F Bader, Power allocation with interference constraint in multicarrier based cognitive radio systems, in Proc (7th International Workshop on MultiCarrier Systems and Solutions (MCSS '09) (Herrsching, Germany, 2009)

29. J Jang, KB Lee, Transmit power adaptation for multiuser OFDM systems. IEEE J Selected Areas Commun 21(2), 171-178 (2003)

30. D Kivanc, G Li, H Liu, Computationally efficient bandwidth allocation and power control for OFDMA. IEEE Trans Wirel Commun 2(6), 1150-1158 (2003)

31. Z Shen, JG Andrews, BL Evans, Optimal power allocation in multiuser OFDM systems, in Proc (IEEE Global Telecommunications Conference (GLOBECOM '03) (San Francisco, USA, 2003)

32. CY Wong, RS Cheng, KB Letaief, RD Murch, Multiuser OFDM with adaptive subcarrier, bit, and power allocation. IEEE J Selected Areas Commun 17(10), 1747-1758 (1999)

33. C Rapp, Effects of the HPA-nonlinearity on 4-DPSK OFDM signal for a digital sound broadcasting system, in Proc Conf. Rec. ECSC'91 (Luettich, Germany, 1991)

34. J Cioffi, Digital Communication: Signal Processing (Standford, California, USA 2000)

35. Q Lu, T Peng, W Wang, Efficient multiuser water-filling algorithm under interference temperature constraints in OFDMA-based cognitive radio networks, in Proc IEEE International Symposium Microwave, Antenna, Propagation, and EMC Technologies for Wireless Communications (MAPE'07, Hangzhou, China, 2007)

36. V Stavroulaki, K Tsagkaris, P Demestichas, J Gebert, M Mueck, A Schmidt, R Ferrus, O Sallent, M Filo, C Mouton, L Rakotoharison, Cognitive control channels: from concept to identification of implementation options. IEEE Commun Mag 50(7), 96-108 (2012)

37. AA Eltholth, AR Mekhail, A Elshirbini, MI Dessouki, Al Abdelfattah, Modeling the effect of clipping and power amplifier non-linearities on OFDM systems. Ubiquitous Comput Commun J 3(1), 54-59 (2009)

38. PHYDYAS, Physical layer for dynamic spectrum access and cognitive radio. http://www.ict-phydyas.org/. Accessed 5 June 2013

39. T Yucek, H Arslan, Spectrum characterization for opportunistic cognitive radio systems, in Proc (IEEE Military Communication Conference, Washington, D.C., USA, 2006)

40. R Jain, Channel Models, A Tutorial (2007). http://www.cse.wustl.edu/ jain/ cse574-08/ftp/channel_model_tutorial.pdf. Accessed 5 July 2013

doi:10.1186/1687-6180-2014-68

Cite this article as: Dikmese et al.: Spectrum sensing and resource allocation for multicarrier cognitive radio systems under interference and power constraints. EURASIP Journal on Advances in Signal Processing 2014 2014:68.

\section{Submit your manuscript to a SpringerOpen ${ }^{\circ}$ journal and benefit from:}

- Convenient online submission

- Rigorous peer review

- Immediate publication on acceptance

- Open access: articles freely available online

- High visibility within the field

- Retaining the copyright to your article

Submit your next manuscript at $>$ springeropen.com 